\title{
Pediatric Intracranial Ependymomas: Prognostic Relevance of Histological, Immunohistochemical, and Flow Cytometric Factors
}

\author{
Josef Zamecnik, M.D., Matija Snuderl, Tomas Eckschlager, M.D., Ph.D., Marketa Chanova, M.D., \\ Marie Hladikova, Michal Tichy, M.D., Roman Kodet, M.D., Ph.D. \\ Department of Pathology and Molecular Medicine (JZ, MS, RK), Department of Pediatric Oncology (TE, \\ $M C)$, Department of Medical Informatics (MH), and Department of Neurosurgery (MT), Charles \\ University, 2nd Medical School, Prague, Czech Republic
}

The correlation between the histological features and clinical outcome remains poor in pediatric intracranial ependymomas. We performed a retrospective study of a group of 31 patients (diagnosed from 1985 to 1995) to assess prognostic implications of the current grading system, of histological and immunohistochemical features, and of ploidy status estimated by flow cytometry. Immunoexpression of a broad spectrum of antigens was evaluated, including MIB-1, topoisomerase-II $\alpha$, cyclin D1, glial and epithelial proteins (GFAP, EMA, cytokeratins), molecules involved in controlling apoptosis (bcl-2, caspase-3/CPP32), and p53 oncoprotein. Univariate and multivariate statistical analyses were performed to evaluate the influence of each variable on both the progression free survival (PFS) and the overall survival (OS) with at least 7-year follow up. Although we showed a significant correlation between histological grade and prognosis, the current grading system failed in predicting outcome in nearly one third of individual cases. Problems with interpathologist reproducibility were also demonstrated. The extent of surgical resection was the only clinical factor that was associated with survival. Both the PFS and the OS were significantly decreased for the following pathological variables: increased cellularity ( $>300$ nuclei per HPF), mitotic activity of $>7$ per $10 \mathrm{HPF}$, increased MIB-1 labeling index (LI), topoisomerase-II $\alpha \mathrm{LI}, \mathrm{S}$-phase fraction,

Copyright (C) 2003 by The United States and Canadian Academy of Pathology, Inc.

VOL. 16, NO. 10, P. 980, 2003 Printed in the U.S.A.

Date of acceptance: June 16, 2003

Supported in part by the following grant sponsors: Ministry of Health of Czech Republic, grant No. VZ FNM 00000064203; Grant Agency of Czech Republic, grant No. GACR 301/001394; Ministry of Education of Czech Republic, grants No. VZ 111300005 and No. VZ J13/98:111300004. Address reprint requests to: Josef Zamecnik, M.D., Department of Pathology and Molecular Medicine, Charles University, 2nd Medical School, V Uvalu 84, 15006 Prague, Czech Republic; fax: 420-224-435-620; e-mail: josef.zamecnik@lfmotol.cuni.cz.

DOI: $10.1097 / 01 . M P .0000087420 .34166 . B 6$ and p53 and bcl-2 positivity. Increased cyclin D1 LI was demonstrated to have only a marginally significant impact on PFS. A flow chart modeling was further performed to formulate a scheme for discriminating of prognostic subgroups. Based on that, p53 immunopositivity and/or MIB-1 LI of $>5 \%$ (after subtotal resection) or MIB-1 LI of $>15 \%$ (after complete resection) were the strongest indicators of the tumor's aggressive behavior and of a poor prognosis of the disease. Foci of hypercellularity should be specifically looked for in ependymomas for assessing the immunohistochemical studies.

KEY WORDS: Bcl-2, DNA flow cytometry, Ependymoma, Grading, MIB-1, p53, Prognosis.

Mod Pathol 2003;16(10):980-991

Ependymomas constitute $6-12 \%$ of all pediatric intracranial malignancies (1). However, the correlation between the histopathologic diagnosis and clinical outcome remains poor (2-6), and the management of patients with ependymomas represents one of the most controversial issues in pediatric oncology.

The recent World Health Organization (WHO) grading system (1) recognizes four ependymal tumor types: subependymoma and myxopapillary ependymoma (Grade I tumors), low-grade ependymoma (Grade II), and anaplastic ependymoma (Grade III). Grade I tumors are clinically and pathologically well-recognized entities with a favorable outcome in most of the patients $(1,7)$. On the other hand, it is difficult to achieve a consensus in discriminating between Grade II and III ependymomas (6). The criteria for distinguishing anaplastic ependymomas defined by WHO grading system (1) remains elusive: ependymal tumors showing increased cellularity and brisk mitotic activity, often accompanied by microvascular proliferation and necrosis and often appearing poorly differentiated. 
The value of individual characteristics as well as their combination necessary to establish the diagnosis has not been settled, which makes the recognition of the anaplastic variant neither easy nor reproducible. Thus, the biologic behavior of ependymomas seems to be difficult to predict on the basis of microscopic appearance alone. Further understanding of molecular invents involved in controlling the tumor growth and their diagnostic detection could lead to establishment of more specific prognostic factors. Antibodies suitable for application on paraffin-embedded archival material have recently allowed this issue to be addressed.

We performed a retrospective study of a group of 31 patients with a follow-up period that was adequate to a slow growth and relatively late recurrence of these tumors. We restricted the study to ependymomas with intracranial location and of the cellular subtype in a cohort of patients who were aged $<18$ years. Both univariate and multivariate statistical analyses were performed to assess prognostic implications of WHO grade, histopathological and immunohistochemical features, and ploidy status. Immunoexpression of a broad spectrum of tumor-associated antigens was evaluated, including molecules involved in cell progression through the cell cycle (MIB-1, topoisomerase-II $\alpha$, cyclin D1), structural glial and epithelial proteins (glial fibrillary acidic protein, epithelial membrane antigen, cytokeratins), molecules involved in controlling apoptosis (bcl-2, caspase-3/CPP32), and p53 oncoprotein. All the data that were obtained in the present series were compared with those investigating similar subjects to make an attempt to design a useful diagnostic and prognostic scheme for childhood ependymomas.

\section{MATERIALS AND METHODS}

Cases included in the study were retrieved from the Pediatric Tumor Registry of the Department of Pathology and Molecular Medicine, Charles University, 2nd Medical School, Prague, Czech Republic. Specimens were obtained from all children (aged $<18$ years) who underwent surgery for newly diagnosed intracranial ependymomas over a 10year period extending from 1985 to 1995 . Children with subependymomas, myxopapillary ependymomas, ependymomas of rare histological subtypes (papillary and clear cell ependymomas), and ependymomas of the spinal cord were excluded because they seem to have a significantly different clinical outcome $(1,8,9)$. The final number of patients enrolled on the study was 31 . The specific histological grade reported at the time of diagnosis was not taken into account. Only the original biopsy specimens with an adequate sampling were used in the study.

\section{Patient Characteristics}

The medical records of each patient were reviewed in detail. The following features were recorded: date of birth, gender, age at the time of surgery, intracranial tumor site, date of surgery for the primary tumor resection, the extent of the resection, the use of adjuvant therapy, and the date of clinically manifested tumor recurrence. Mean age of the patients at the time of diagnosis was 5.8 years, ranging from 11 months to 14 years. There were 19 boys and 12 girls. The tumor location was supratentorial in 16 patients and was infratentorial in 15 patients. The extent of resection was assessed based on both the operative notes and the early postoperative imaging. Anything less than a gross total resection was considered a subtotal resection. None of the patients died from surgical complications or in the early postoperative period. The tumor removal was complete in nine patients. Twenty-five patients underwent radiotherapy, and 23 patients received chemotherapy; 22 of them received both the chemotherapy and radiotherapy. Doses of local postoperative irradiation varied from 30 to 52 Gy according to the patients' age. Progression-free survival (PFS) was defined as the time from the initial surgery to the date of evidence of the tumor progression confirmed radiologically. Overall survival (OS) was calculated as the time from surgery to death, or as the time to the last follow-up appointment of the surviving patients.

\section{Pathological Analysis}

All specimens were fixed in $10 \%$ neutral buffered formalin and paraffin embedded. Fourmicrometer-thick tissue sections were recut from paraffin blocks for routine hematoxylin and eosin staining. Before evaluating clinical data that were obtained from the study and exact quantification of appropriate variables, the histological grade according to the WHO classification criteria (1) was evaluated independently by three observers (JZ, RK, MS) to simulate the routine diagnostic process. Presence of increased cellularity, vascular proliferation, nuclear polymorphism, necrosis, and count of mitotic figures were taken into consideration. Because the presence of necrosis in ependymal tumors was repeatedly shown to have only a minor, if any, impact in assessing their biological behavior $(10,11)$, the presence of necrosis alone did not allow the diagnosis of anaplastic ependymoma in the present study. We graded each sample as an anaplastic (Grade III) or as a low-grade (Grade II) ependymoma when consensus between at least two of the observers was achieved.

A further seven histological parameters (the presence of hypercellular nodules, collagen production, bone and cartilage, calcification, psammoma bod- 
ies, thrombi) were judged as present or absent in each case independently. The presence of perivascular pseudorosettes and true ependymal rosettes was also noted. Necrosis (with or without pseudopalisading) was considered to be present when small or large areas of coagulation-type necrosis were seen. Isolated necrotic cells were not considered significant. Vascular proliferation was defined as hyperplasia of endothelial cells forming multilayered small vessels.

\section{Immunohistochemistry}

We selected a representative paraffin block from each case, and the block was recut for immunohistochemical staining purposes. Four-micrometerthick tissue sections were deparaffinized in xylene and rehydrated through decreasing concentrations of ethanol to water. We used the following monoclonal antibodies:

1. Ki-67 (clone MIB-1, 1:100, microwave pretreatment; DAKO Co.).

2. Phospho-topoisomerase II-alpha (topo-II $\alpha$, clone PT/3D4, 1:100; Immunotech Co.).

3. Cyclin D1 (clone DCS-6, 1:1600, enzyme predigestion and microwave pretreatment; DAKO Co.).

4. p53 (clone DO-7, 1:100, microwave pretreatment; DAKO Co.).

5. Bcl-2 (clone 124, 1:40; DAKO Co.).

6. Caspase-3/CPP32 (clone 19, prediluted, microwave pretreatment; Immunotech Co.).

7. Glial fibrillary acidic protein (GFAP; clone 6F2, 1:1000, microwave pretreatment; DAKO Co.).

8. Epithelial membrane antigen (EMA; clone E29, 1:100, microwave pretreatment; DAKO Co.).

9. Cytokeratin (CK; clone AE1/AE3, 1:200, enzyme predigestion; DAKO Co.).

The sections were incubated overnight at $4^{\circ} \mathrm{C}$ with appropriate primary antibodies. Except for anti-cyclin D1 assay, the antigen-antibody complexes were visualized by biotin-streptavidin detection system (DAKO ChemMate Detection kit) with 3,3'-diaminobenzidine (DAB Fluka Chemie GmbH.) as chromogen. Supersensitive detection system (Biogenex; cat. no. ZA000-UM) with alkaline phosphatase-conjugated streptavidin with fast red (Biogenex; cat. no. HK 182-5K) was used for immunolocalization of cyclin D1 epitope. Positive and negative controls were provided with each assay.

Each slide was studied under high-power magnification (high-power field, HPF; 400×, area comprising $0.017 \mathrm{~mm}^{2}$ ). The images were computerized and manually counted. For each evaluated variable, $\geq 1000$ cells in at least five HPFs were counted. Blood-derived cells and vascular components were excluded from the analysis, and the counting procedure was performed in viable regions of the tumors only. The regions having the greatest number of immunoreactive cells were chosen for counting the immunohistochemical variables.

Mitotic activity was obtained as the single highest mitotic count of $>20$ consecutive HPFs and was expressed as a mean value for 10 HPFs. After subjective evaluating of increased cellularity (present or absent) by all observers, the cell density was exactly counted in 20 consecutive HPFs and expressed as the mean value for one HPF.

Anti-GFAP stained the cell processes of perivascular pseudorosettes. Granular immunoreactive product with anti-EMA was present in the peripheral aspects of the tumor cells. Intracytoplasmic reaction products in the tumor cells were judged as positive in reaction with anti-CK, anti-bcl-2, and anti-CPP32. Staining for p53 was considered positive if distinct nuclear staining (regardless the intensity) was present. After counting, the results of p53, bcl-2, GFAP, CK, and EMA immunoreactions were expressed semiquantitatively (absent or present). To exclude evaluation of equivocal reactions the staining was considered positive if $>1 \%$ of the neoplastic cells were stained.

Results of immunoreactions with antibodies directed against MIB-1, topo-II $\alpha$, and cyclin D1 antigens were expressed by their labeling indices (LIs), defined as the percentage of immunoreactive nuclei divided by the total number of the tumor cells in the evaluated areas.

\section{DNA Flow Cytometry}

For DNA flow cytometric analysis, paraffinembedded tissue blocks were processed by the modified Hedley's method (12). Fifty-micrometerthick tissue sections, which were prepared from nonnecrotic parts of the tumors, were deparaffinized in xylene and rehydrated by decreasing concentration of ethanol to water. Nuclear suspensions were prepared by incubation in $0.25 \%$ pepsin solution in diluted $\mathrm{HCl}$. Nuclei were stained by propidium iodide using the DNA Prep Reagent kit (Coulter Immunology, Hialeah, FL) according to the manufacturer's protocol. The kit contains permeabilization solution and staining solution: propidium iodide with RNase. After the staining procedure, the samples were measured with FACSCalibur cytometer (Beckton Dickinson, San Jose, CA), and the data were analyzed by software ModFit LT (Verity Software House, Inc., Topsham, ME). The DNA content of the aneuploid tumor cells is expressed as the DNA index (DI) ratio of the modal G0/G1 peak of the aneuploid cells to the modal G0/G1 peak of the diploid cells in a sample. Percentages (fractions) of cells in G0/G1, S, and G2/M phases of the cell cycle were also evaluated. 


\section{Statistical Methods}

Five-year OS and PFS were the primary endpoints of this analysis. The PFS and OS curves were plotted using the Kaplan-Meier method. Univariate analysis was performed using a log-rank test to assess the strength of association between all subgroups of patients and the outcome. Relative risks (hazard ratios) were computed using univariate and multivariate Cox proportional hazards regression analysis. In the multivariate model, the variables were statistically selected by forward stepwise inclusion. Classification and regression tree (CART) analysis was performed to identify cutpoints and prognostic subgroups with most significant difference in the clinical outcome. Associations between categorical variables were assessed via the Pearson's $\chi^{2}$ test, associations between numeric variables were assessed via Spearman's rank correlation analysis (with correlation coefficient estimate, $r$ ), and associations between categorical and numeric variables were assessed via the Mann-Whitney test. The analytical work was performed using SPSS (Version 10, SPSS Inc.) software. Probability $(P)$ values of $<.05$ were considered significant or highly significant when $P<.01$. A 95\% confidence interval was used.

\section{RESULTS}

Based on histopathological findings from a total of 31 cases, 11 were evaluated as low grade (Grade II), and 20 as anaplastic (Grade III) ependymomas. In 21 cases $(67.7 \%)$, consensus concerning the grade was reached by all three observers; in the remaining 10 cases $(32.3 \%)$, two observers only were in agreement.

The median PFS for the entire cohort of patients was 29 months, and the 5-year OS rate was $45.2 \%$. The follow-up revealed that $14(45.2 \%)$ were alive with no evidence of the disease within the period ranging from 73 to 176 months after the operation (mean, 135 mo; median, 156 mo). Local tumor recurrence developed in 17 (54.8\%) cases with PFS times varying from 2 to 58 months (mean, 17.2 mo; median, $13 \mathrm{mo}$ ). All of these patients died of tumor with the interval between the surgery and death ranging from 2 to 61 months (mean, 21.8 mo; median, $17 \mathrm{mo}$ ). Death occurred within an interval of 1 to 22 months (mean, 4.7 mo; median, 3 mo) after the tumor recurrence. The 5-year OS of patients with recurrent tumors was only $6 \%$. Recurrences were encountered in $27.3 \%$ and $70 \%$ of low-grade and anaplastic ependymomas, respectively. Both the median PFS and the 5-year OS were significantly reduced for anaplastic tumors (log-rank test, $P=.02$ and .03 , respectively).

Clinical variables and their distribution between low-grade and anaplastic ependymomas are sum- marized in Table 1A. Tumor site, age, or gender of the patients did not reveal a significant prevalence in the studied subgroups of patients.

\section{Histopathology}

By light microscopy, all examined tumors were characterized by perivascular pseudorosettes and presented histopathological features typical of "classic" cellular ependymoma. Histological findings are summarized in Table 1B.

Increased mitotic activity, increased number of nuclei per HPF, and vascular proliferations were significantly prevalent in the group of anaplastic ependymomas. Subjective evaluation of increased cellularity corresponded in all observers with exact counted values of cell density in 23 cases $(74.2 \%)$ in categories $<250$ (designed as absent hypercellularity) and $>370$ (designed as present hypercellularity) cells per HPF. In eight cases (25.8\%) of intermediate cellularity (250-370 cells per HPF), the consensus was not reached. In two low-grade tumors (18.2\%) and in nine anaplastic tumors (45\%), the focal presence of hypercellular nodules was observed. All tumors with such foci recurred within 7 to 34 months (mean, $17.1 \mathrm{mo}$; median, $18 \mathrm{mo}$ ), and the patients died of the tumor. Lymphocytes, thrombi, collagen production, calcification, bone and cartilage, or psammoma bodies were noted only sporadically in both subgroups of tumors without an evident link to the anaplastic features, histopathological grade, or clinical outcome (data not shown).

\section{Immunohistochemistry}

Results of immunohistochemical studies are summarized in Table 1C.

All ependymomas exhibited nuclear Ki-67 and topo-II $\alpha$ accumulation. Anaplastic tumors revealed more prominent labeling indices of MIB-1 (medians, $12.2 \%$ versus $1.2 \%, P<.0005$ ). Two patterns of topo-II $\alpha$ nuclear positivity were observed, the weak and the strong one. Topo-II $\alpha$ LI based on counting of all positive cells varied from 1.7 to $98.4 \%$ (median, $37.1 \%$ ). Such topo-II $\alpha$ LI correlated not with mitotic activity, MIB-1 LI, histopathological grade, nor with proliferative fractions evaluated by flow cytometric study. Topo-II $\alpha$ LI based on counting of strongly positive nuclei only correlated significantly with all above-mentioned criteria $(P<.0005)$, ranging from 0.7 to $12.7 \%$ in low-grade tumors (median, $2.6 \%$ ) and from 3.5 to $38.6 \%$ for anaplastic tumors (median, 16.7\%). Of 31 cases, 20 showed nuclear positivity with anti-cyclin D1 antibody, with the percentage of positive cells ranging from 0.2 to $97.2 \%$. The increased cyclin D1 LI was not significantly prominent in anaplastic tumors. 
TABLE 1. Summary of Clinical (A), Histological (B), Immunohistochemical (C) and Flow Cytometric (D) Variables and Their Distribution between Grade II and Grade III Ependymomas

\begin{tabular}{|c|c|c|c|c|}
\hline & Total & Grade II & Grade III & $\begin{array}{l}\text { Statistical Comparison } \\
\text { between Grade II and III }\end{array}$ \\
\hline Cases (\% of total) & 31 & $11(35.5 \%)$ & $20(64.5 \%)$ & - \\
\hline \multicolumn{5}{|l|}{ A. } \\
\hline Median PFS (months)/range/ & $29 / 2-176 /$ & $97 / 8-174 / *$ & $18 / 2-176 /$ & $\operatorname{lr} ; P=0.02$ \\
\hline 5 -year OS & $14(45.2 \%)$ & 8 (72.7\%)* & $6(30 \%)$ & $\operatorname{lr} ; P=0.03$ \\
\hline Recurrent tumors & $17(54.8 \%)$ & $3(27.3 \%)$ & $14(70.0 \%)^{*}$ & chi; $P=0.03$ \\
\hline Mean age (years)/range/ & $5.8 / 1-14 /$ & $5.3 / 1-13 /$ & $6.1 / 1-14 /$ & mw; NS \\
\hline Male gender & $19(61.3 \%)$ & $7(63.6 \%)$ & $12(60 \%)$ & chi; NS \\
\hline Supratentorial site & $16(51.6 \%)$ & $5(45.5 \%)$ & $11(55 \%)$ & chi; NS \\
\hline Subtotal resection & $22(71.0 \%)$ & $9(81.8 \%)$ & $13(65 \%)$ & chi; NS \\
\hline \multicolumn{5}{|l|}{ B. } \\
\hline Median cell count-1 HPF/range/ & $368 / 119-547 /$ & $184 / 119-399 /$ & $439 / 202-547 / *$ & mw; $P=0.0001$ \\
\hline Median mitoses per $10 \mathrm{HPF} /$ range/ & $7 / 0-28 /$ & $2 / 0-4 /$ & $13 / 5-28 / *$ & mw; $P<0.0005$ \\
\hline Necrosis & $18(58.1 \%)$ & $4(36.4 \%)$ & $14(70.0 \%)$ & chi; NS \\
\hline Vascular proliferation & $19(61.3 \%)$ & $3(27.3 \%)$ & $16(80.0 \%)^{*}$ & chi; $P=0.007$ \\
\hline Nuclear pleomorphism & $18(58.1 \%)$ & $4(36.4 \%)$ & $14(70.0 \%)$ & chi; NS \\
\hline Hypercellular nodules & $11(35.5 \%)$ & $2(18.2 \%)$ & $9(45.0 \%)$ & chi; NS \\
\hline \multicolumn{5}{|l|}{ C. } \\
\hline Median MIB-1 LI (\%)/range/ & $9.7 / 0.4-34.3 /$ & $1.2 / 0.4-9.0 /$ & $12.2 / 2.0-34.3 / *$ & mw; $P<0.0005$ \\
\hline Median topo-II $\alpha$ LI (\%)/range/ & $12.7 / 0.7-38.6 /$ & $2.6 / 0.7-12.7 /$ & $16.7 / 3.5-38.6 / *$ & $\mathrm{mw} ; P<0.0005$ \\
\hline Median cyclin D1 LI (\%)/range/ & $1.6 / 0-97.2 /$ & $0.2 / 0-8.4 /$ & $2.9 / 0-97.2 /$ & mw; NS \\
\hline p53 positive & $11(35.5 \%)$ & $1(9.1 \%)$ & $10(50.0 \%)^{*}$ & chi; $P=0.047$ \\
\hline bcl-2 positive & $14(45.2 \%)$ & $3(27.3 \%)$ & $11(55.0 \%)$ & chi; NS \\
\hline Caspase-3/СРР32 positive & $4(12.9 \%)$ & $2(18.2 \%)$ & $2(10.0 \%)$ & chi; NS \\
\hline GFAP positive & $24(77.4 \%)$ & $10(90.9 \%)$ & $14(70.0 \%)$ & chi; NS \\
\hline EMA positive & $6(19.4 \%)$ & $3(27.3 \%)$ & $3(15.0 \%)$ & chi; NS \\
\hline \multicolumn{5}{|l|}{ D. } \\
\hline Aneuploid & $14(46.7 \%)$ & $3(27.3 \%)$ & $11(57.9 \%)$ & chi; NS \\
\hline Median S-phase fraction (\%)/range/ & $3.4 / 0-23.9 /$ & $1.1 / 0.1-4.7 /$ & $4.4 / 0-23.9 / *$ & $\mathrm{mw} ; P=0.007$ \\
\hline Median S+G2/M-phase fraction (\%)/range/ & $6.2 / 1.4-33.3 /$ & $3.4 / 1.4-33.3 /$ & $7.4 / 1.6-27.3 /^{*}$ & mw; $P=0.017$ \\
\hline
\end{tabular}

LI: labeling index; topo-II $\alpha$ : phospho-topoisomerase-II $\alpha$; HPF: high power field; lr: log-rank test; chi: chi-square test; mw: Mann-Whitney test; NS: not significant; * variable significantly prevalent in a subgroup.

The nuclear positivity of p53 protein was observed in $11(35.5 \%)$ cases and was marginally significantly prevalent in anaplastic tumors $(P=.047$; Fig. 1). All except one tumor overexpressing p53 protein were characterized by recurrences within 2 to 34 months (mean, 16.1 mo; median, 14 mo). The patient without recurrence of p53-positive ependymoma had a complete surgical resection. Bcl-2 protein-positive tumors did not reach a significant

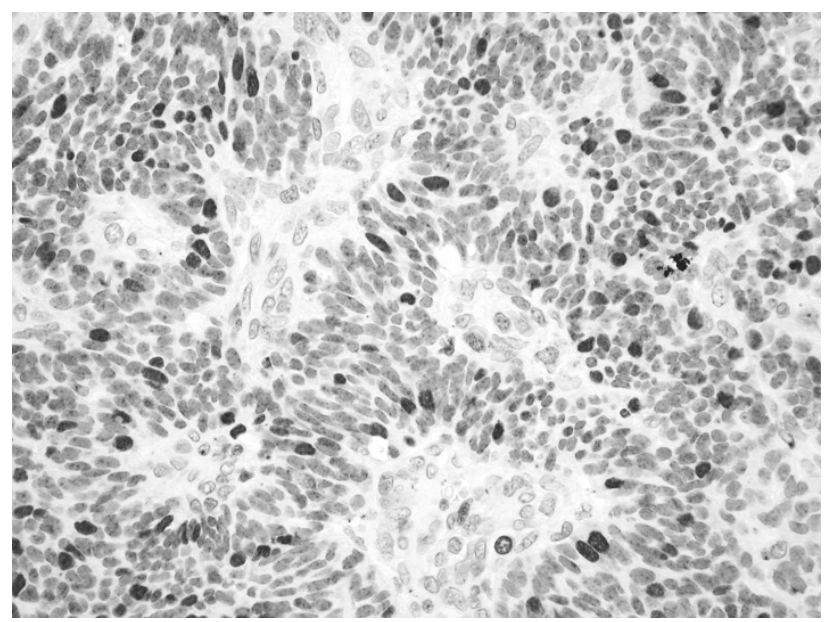

FIGURE 1. A strong nuclear immunoreactivity of an ependymoma for p53 protein (immunoperoxidase method, counterstained lightly with hematoxylin; original magnification, $400 \times$ ). prevalence in anaplastic ependymomas (Fig. 2). From 14 bcl-2-positive tumors, 11 (78.6\%) had a recurrence within the period of 2 to 34 months (mean, 14.6 mo; median, 13 mo), and these patients died of the tumor. Eight of 14 bcl-2-positive cases overexpressed simultaneously p53 protein; in 5 of these cases, we found hypercellular nodules, in which both the p53, MIB-1, and bcl-2 immunoreactions were strongly expressed (Fig. 3). Weak focal

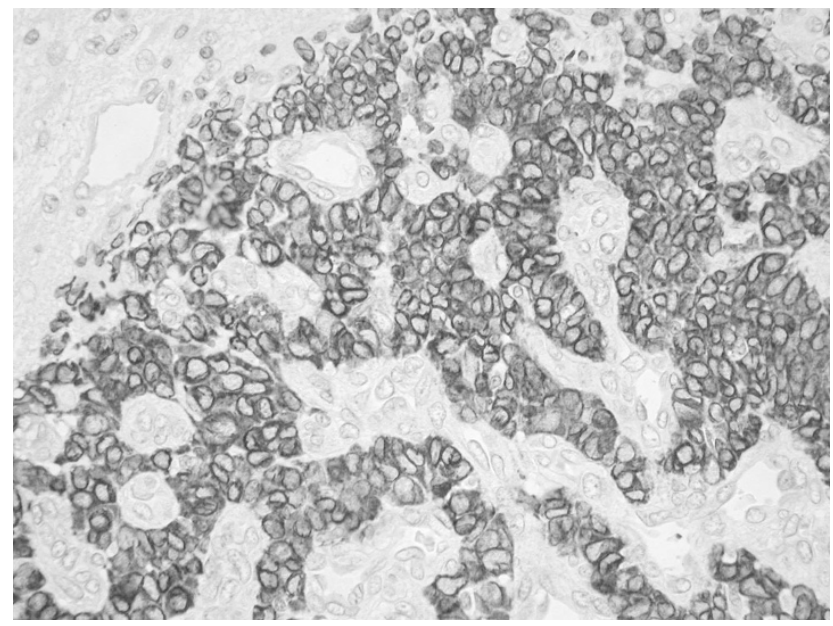

FIGURE 2. Diffuse cytoplasmic immunoreactivity of an ependymoma for bcl-2 protein (immunoperoxidase method, counterstained lightly with hematoxylin; original magnification, $400 \times$ ). 
immunoexpression of caspase-3/CPP32 was observed in four tumors (12.9\%), two cases from both the low-grade and anaplastic group. Immunoprecipitate was localized not only in the cytoplasm of the tumor cells, but also in the cytoplasm of endothelial cells lining proliferating vessels. Except for one case, the tumors were simultaneously caspase3/CPP32 positive and bcl-2 negative. None of the tumors reacted with anti-cytokeratin antibodies. Immunoexpression of GFAP and EMA were not significantly associated with either grade of ependymomas.

\section{DNA Flow Cytometry}

The results of the flow cytometric studies are summarized in Table 1D.

The DNA analysis exhibited 16 diploid tumors; 14 cases were aneuploid with DNA indices ranging from 1.17 to 2.47. In 10 of 16 aneuploid tumors $(62.5 \%)$, a marked nuclear pleomorphism was observed also morphologically. Both the S-phase and $\mathrm{S}+\mathrm{G} 2 / \mathrm{M}$-phase fraction prevailed in anaplastic ependymomas ( $P=.007$ and .017 , respectively). In one case with hypercellular nodules, two obvious G0/G1 peaks (1.3 and 1.5) and two proliferation fractions (3.7 and 3.9 for $S$ phase, 1.7 and 8.2 for $\mathrm{S}+\mathrm{G} 2 / \mathrm{M}$ phase) were observed (Fig. 4). One case was eliminated from DNA flow cytometric studies because of poor-quality histograms.

\section{Correlation Analyses}

Relationships among all combinations of pairs of clinical, pathological, immunohistochemical, and flow cytometric variables were examined. Statistical analysis of correlations of six variables involving tumor cell proliferation kinetics (mitotic activity, MIB-1 LI, topo-II $\alpha$ LI, cyclin D1-LI, S-phase, and $\mathrm{S}+\mathrm{G} 2 / \mathrm{M}$-phase fractions) and cell density is out-

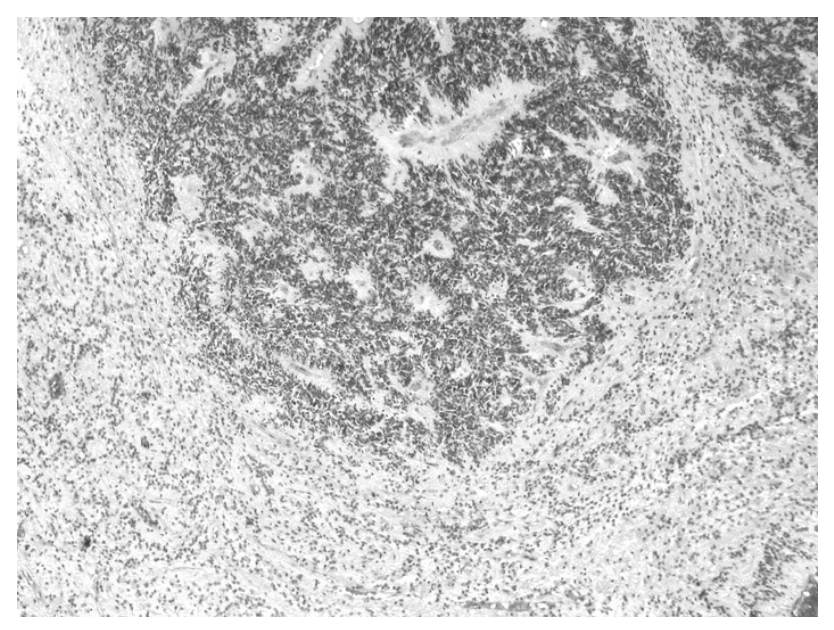

FIGURE 3. A hypercellular focus in an ependymoma of an otherwise low tumor cell density (hematoxylin-eosin; original magnification, $40 \times$ ).

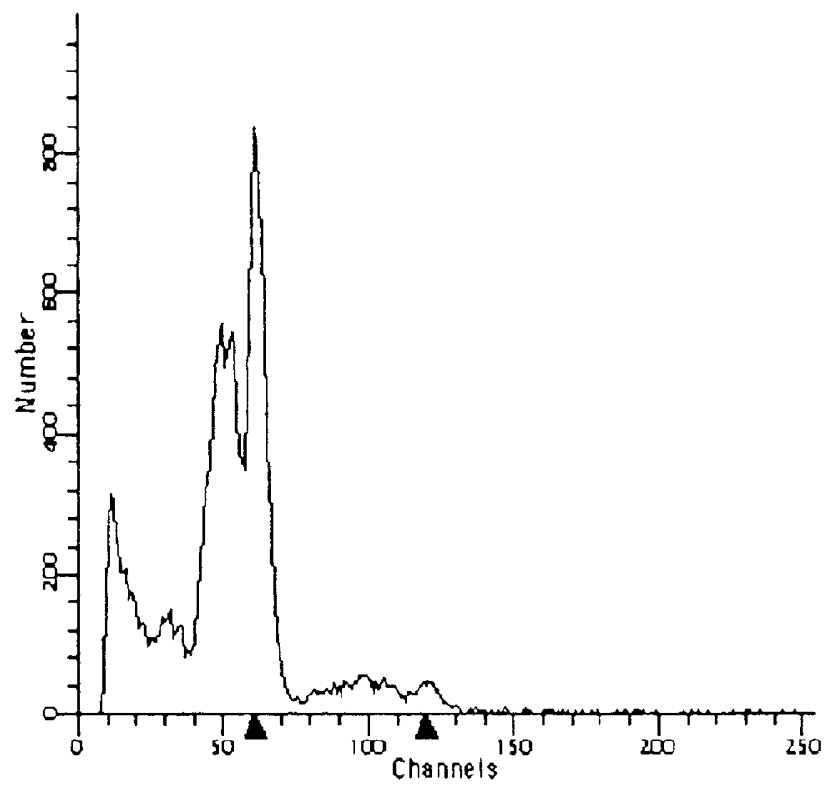

FIGURE 4. DNA histogram. Two obvious G0/G1 peaks (DNA contents 1.3 and 1.5) in an ependymoma with hypercellular nodules observed by histology.

lined in Table 2. Only cyclin D1-LI did not reveal a significant correlation with any of the variables. Beside that, expression of both p53 and bcl-2 was significantly associated with an increased proliferative activity of the tumor cells (Mann-Whitney tests; in each pair, $P<.01$ ). Presence of necrosis was associated with vascular proliferation and pleomorphism ( $\chi^{2}$; $P$ values .003 and .0005 , respectively). Pleomorphism was strongly related to aneuploid status of the tumor $\left(\chi^{2} ; P=.03\right)$. No other significant correlation was demonstrated.

\section{Survival and CART Analyses}

Using CART analysis, age of $<4$ years, cell density of $>300$ cells per HPF, mitotic activity of more than seven mitoses per $10 \mathrm{HPFs}$, MIB-1 LI of $>7 \%$, topoII $\alpha$ LI of $>12 \%$, cyclin D1 LI of $>5 \%$, S-phase fraction of $>3 \%$, and $\mathrm{S}+\mathrm{G} 2 / \mathrm{M}$-phase fraction of $>5 \%$ were most significant for a decrease in survival, and the corresponding values were designed as cut-off points. Values for p53, bcl-2, caspase-3/CPP32, EMA, GFAP, and the remaining histological factors were grouped as negative versus positive.

The results of univariate survival analysis (log rank testing and Cox proportional hazards regression analysis) are shown in Table 3 and Figure 5. The histological grade was shown to correlate significantly with clinical outcome. The extent of surgical resection was the only clinical factor that was strongly associated with survival. For the whole cohort of patients, both the PFS and the OS were further significantly decreased for subgroups of increased cellularity, presence of hypercellular nod- 
TABLE 2. Statistical Correlation among Variables Displaying Proliferative Activity of Tumor Cells (Spearman's $r$ Correlation Coefficient)

\begin{tabular}{|c|c|c|c|c|c|c|}
\hline & Mitotic activity & & & & & \\
\hline MIB-1 LI & $\begin{array}{l}\mathrm{r}=0.87 \\
P<0.0005^{* *}\end{array}$ & MIB-1 LI & & & & \\
\hline Topo-II $\alpha$ LI & $\begin{array}{l}\mathrm{r}=0.84 \\
P<0.0005^{* *}\end{array}$ & $\begin{array}{l}\mathrm{r}=0.93 \\
P<0.0005^{* *}\end{array}$ & Topo-II $\alpha$ LI & & & \\
\hline Cyclin D1 LI & $\begin{array}{l}\mathrm{r}=0.34 \\
\mathrm{NS}\end{array}$ & $\begin{array}{l}\mathrm{r}=0.22 \\
\mathrm{NS}\end{array}$ & $\begin{array}{l}\mathrm{r}=0.32 \\
\mathrm{NS}\end{array}$ & Cyclin D1 LI & & \\
\hline S-phase & $\begin{array}{l}\mathrm{r}=0.58 \\
P=0.001^{* *}\end{array}$ & $\begin{array}{l}\mathrm{r}=0.67 \\
P<0.0005^{* *}\end{array}$ & $\begin{array}{l}\mathrm{r}=0.72 \\
P<0.0005^{* *}\end{array}$ & $\begin{array}{l}r=0.19 \\
\mathrm{NS}\end{array}$ & S-phase & \\
\hline $\mathrm{S}+\mathrm{G} 2 / \mathrm{M}$ phase & $\begin{array}{l}\mathrm{r}=0.42 \\
P=0.021^{*}\end{array}$ & $\begin{array}{l}\mathrm{r}=0.46 \\
P=0.01^{*}\end{array}$ & $\begin{array}{l}\mathrm{r}=0.56 \\
P=0.001^{* *}\end{array}$ & $\begin{array}{l}r=0.04 \\
N S\end{array}$ & $\begin{array}{l}\mathrm{r}=0.62 \\
P=0.001^{* *}\end{array}$ & $\mathrm{~S}+\mathrm{G} 2 / \mathrm{M}$ phase \\
\hline Cell density & $\begin{array}{l}\mathrm{r}=0.84 \\
P<0.0005^{* *}\end{array}$ & $\begin{array}{l}\mathrm{r}=0.81 \\
P<0.0005^{* *}\end{array}$ & $\begin{array}{l}\mathrm{r}=0.81 \\
P<0.0005^{* *}\end{array}$ & $\begin{array}{l}\mathrm{r}=0.31 \\
\mathrm{NS}\end{array}$ & $\begin{array}{l}\mathrm{r}=0.57 \\
P=0.001^{* *}\end{array}$ & $\begin{array}{l}\mathrm{r}=0.39 \\
P=0.034^{*}\end{array}$ \\
\hline
\end{tabular}

LI: labeling index; topo-II $\alpha$ : phospho-topoisomerase-II $\alpha$; NS: not significant; ${ }^{*}$ significant, ${ }^{* *}$ highly significant.

TABLE 3. Univariate Analysis of Clinical, Histological, Immunohistochemical and Flow Cytometric Factors by Cox Proportional Hazards Modeling

\begin{tabular}{|c|c|c|c|c|c|}
\hline & \multirow{2}{*}{$\begin{array}{c}\text { Frequency } \\
(\%)\end{array}$} & \multicolumn{2}{|c|}{ PFS } & \multicolumn{2}{|c|}{ OS } \\
\hline & & Risk Ratio (CI) & $P$ & Risk Ratio (CI) & $P$ \\
\hline Grade 3 & 64.5 & $4.1(1.2-14.5)$ & $0.02^{*}$ & $3.8(1.1-13.5)$ & $0.03^{*}$ \\
\hline Age $<4$ years & 35.5 & $2.1(0.8-5.5)$ & NS & $2.3(0.9-6.1)$ & NS \\
\hline Male gender & 61.3 & $1.2(0.4-3.1)$ & NS & $1.2(0.5-3.1)$ & NS \\
\hline Supratentorial site & 51.6 & $1.4(0.5-3.5)$ & NS & $1.3(0.5-3.4)$ & NS \\
\hline Subtotal resection & 71.0 & $5.1(1.2-22.3)$ & $0.015^{*}$ & $5.2(1.2-22.9)$ & $0.017^{*}$ \\
\hline Chemotherapy & 74.2 & $2.1(0.6-9.2)$ & NS & $1.7(0.6-10.3)$ & NS \\
\hline Radiotherapy & 80.6 & $2.5(0.9-9.9)$ & NS & $2.2(0.7-11.3)$ & NS \\
\hline Necrosis & 58.1 & $1.3(0.5-3.4)$ & NS & $1.3(0.5-3.3)$ & NS \\
\hline Vascular proliferation & 61.3 & $1.4(0.5-3.7)$ & NS & $1.3(0.5-3.6)$ & NS \\
\hline Nuclear pleomorphism & 58.1 & $0.9(0.4-2.4)$ & NS & $0.9(0.3-2.3)$ & NS \\
\hline True rosettes absent & 58.1 & $0.9(0.4-2.4)$ & NS & $0.9(0.3-2.4)$ & NS \\
\hline Cell density $>300 / \mathrm{HPF}$ & 71.0 & $24.9(1.8-859.3)$ & $<0.001^{* *}$ & $25.2(1.9-795.4)$ & $<0.001^{* *}$ \\
\hline Hypercellular nodules & 35.5 & $6.4(2.1-19.7)$ & $<0.001^{* *}$ & $7.5(2.3-15.2)$ & $<0.001^{* *}$ \\
\hline Mitoses $>7 / 10 \mathrm{HPF}$ & 48.4 & $8.5(2.7-27.1)$ & $<0.001^{* *}$ & $8.0(2.5-25.4)$ & $<0.001^{* *}$ \\
\hline MIB-1 LI >7\% & 58.1 & $27.3(3.5-212.3)$ & $<0.001^{* *}$ & $27.0(3.5-210.3)$ & $<0.001^{* *}$ \\
\hline Topo-II $\alpha>12 \%$ & 51.6 & $8.5(2.3-30.5)$ & $<0.001^{* *}$ & $8.1(2.2-29.2)$ & $<0.001^{* *}$ \\
\hline Cyclin D1 LI > $\% \%$ & 35.5 & $2.6(1.0-6.9)$ & $0.049 *$ & $2.5(0.9-6.5)$ & NS \\
\hline p53 positive & 35.5 & $6.0(2.0-17.7)$ & $<0.001^{* *}$ & $5.8(1.9-17.3)$ & $0.0015^{* *}$ \\
\hline bcl-2 positive & 45.2 & $5.3(1.8-15.4)$ & $<0.001^{* *}$ & $5.7(1.9-16.6)$ & $<0.001^{* *}$ \\
\hline Caspase3/CPP32 positive & 12.9 & $1.1(0.3-4.9)$ & NS & $1.1(0.3-4.9)$ & NS \\
\hline GFAP negative & 22.6 & $1.7(0.6-4.9)$ & NS & $1.7(0.6-4.9)$ & NS \\
\hline EMA positive & 19.4 & $0.5(0.1-2.2)$ & NS & $2.2(0.5-9.5)$ & NS \\
\hline S-phase $>3 \%$ & 53.3 & $4.1(1.3-12.7)$ & $0.02^{*}$ & $4.0(1.3-12.5)$ & $0.01^{*}$ \\
\hline $\mathrm{S}+\mathrm{G} 2 / \mathrm{M}$ phase $>5 \%$ & 53.3 & $2.3(0.9-6.4)$ & NS & $2.3(0.9-6.4)$ & NS \\
\hline Aneuploid & 46.7 & $1.0(0.4-2.6)$ & NS & $1.0(0.4-2.5)$ & NS \\
\hline
\end{tabular}

PFS: progression-free survival; OS: overall survival; CI: confidence interval; LI: labeling index; HPF: high power field; topo-II $\alpha$ : phospho-topoisomerase-II $\alpha$; NS: not significant; * significant; ** highly significant.

ules, increased mitotic activity, MIB-1 LI, topo-II $\alpha$ LI and S-phase fraction, and with p53 and bcl-2 positivity. Increased cyclin D1 LI was demonstrated to have only a marginally significant impact on PFS $(P=.049)$; its association with OS was not significant.

The multivariate analysis of the entire cohort revealed that decrease of both PFS and OS was significantly associated with a subtotal surgical resection and with MIB-1 LI of $>7 \%$, as indicated in Table 4 .

We built a flow chart of prognostic criteria discriminating between patients with favorable and unfavorable prognosis. Models composed of all possible combinations of the significant parameters in univariate analyses were tried. Cut-off values for continuous variables were re-estimated for each subgroup depending on the extent of tumor removal. The number of parameters in the model was increased step by step until the significance of the log-rank statistic was not further increased. The model having the most significant log-rank statistic was regarded as the final model. The most different survival curves examined by log-rank testing were exhibited by evaluating the extent of resection, together with p53 positivity and MIB-1 LI, with cut-off values of $5 \%$ for subtotal resection and of $15 \%$ for a complete resection, as shown in Table 5 and Figure 6. Risk ratios (CI) of the scheme reached 39.2 (4.9315.0) for a decrease of PFS $(P<.0005)$ and reached $38.8(4.8-311.8)$ for a decrease of OS $(P<.0005)$. 

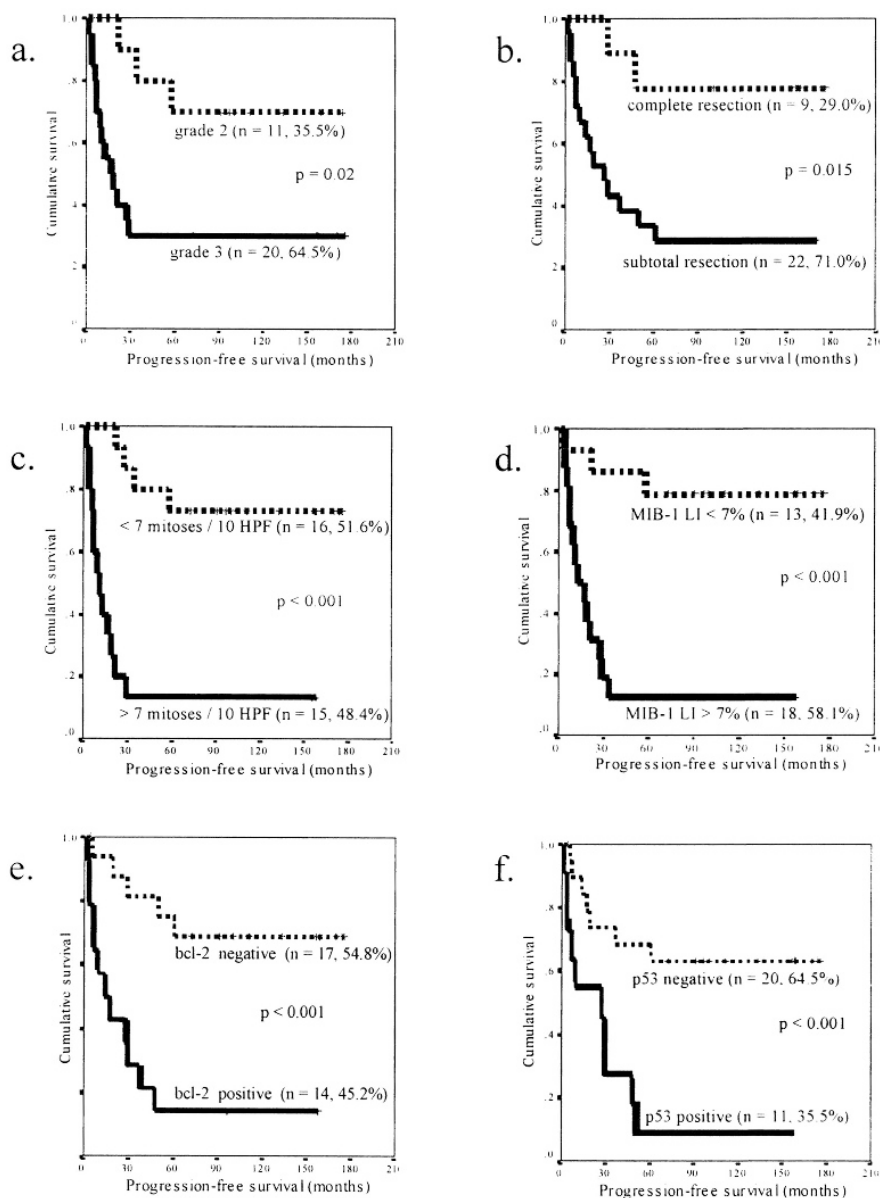

FIGURE 5. Graphs showing representative Kaplan-Meier survival curves of the patients with ependymomas grouped according to the evaluated variables. $P$ values of log-rank testing. A, histopathological grades; B, extent of resection; C, mitotic activity; D, MIB-1 labeling index; E, bcl-2 immunoreactivity; and F, p53 immunoreactivity.

\section{DISCUSSION}

The prognosis of patients with ependymomas remains poor. The recurrence rate of $54.8 \%$ in the present study within the period of follow-up from 73 to 176 months and the 5-year survival of $45.2 \%$ are in concordance with data obtained in reported studies of pediatric intracranial ependymomas (6, $7,13,14)$.

None of the clinical factors, such as the primary site of the tumor in the brain or gender of the patient, achieved significance in predicting the clinical outcome in previously published series (3, $5,10,13,15)$, as is the case with our study. Although a reduced PFS or OS in children in lower age categories was observed by some investigators $(5,14$, $16,17)$, it was not significant in our series. In our statistical analyses, the extent of the tumor resection was the only clinical variable that was strongly linked with both the reduced PFS and 5-year OS. A significant decrease of the recurrence rate was also observed after a complete removal of the primary tumor by other observers $(3,5,10,15,16,18)$. There remain many unanswered aspects concerning the effectiveness of both chemotherapy and radiotherapy (reviewed by Bouffet et al. (6)), and no beneficial effect of adjuvant therapies was evident in our series.

In contrast to astrocytic neoplasms, in which traditional histological markers of aggressiveness allow consistent prognostic assessment, correlation of histopathology with the outcome in ependymomas has been notoriously questionable (6). Some investigators found a statistically significant relevance of histopathological grade for a long-term prognosis $(13,14,16,19)$, whereas others did not (2, $3,5,18,20)$. Our study demonstrated a statistical significance of correlation between histological grade and prognosis. However, $27.3 \%$ of patients with histologically determined low-grade lesions died of tumor, in contrast to $30.0 \%$ of patients with anaplastic lesions who were alive and without evidence of the tumor $\geq 7$ years after the surgery. Thus the histological grading failed in predicting outcome in nearly one third of individual cases. The problematic reproducibility of the current grading scheme was illustrated by the lack of interpersonal 
TABLE 4. Multivariate Analysis of Progression-Free Survival and Overall Survival by Cox Proportional Hazards Modeling

\begin{tabular}{|c|c|c|c|c|}
\hline & \multicolumn{2}{|c|}{ PFS } & \multicolumn{2}{|c|}{ OS } \\
\hline & Risk ratio (CI) & $P$ & Risk ratio (CI) & $P$ \\
\hline Subtotal resection & $5.9(1.4-25.7)$ & $0.017^{*}$ & $7.6(1.6-35.2)$ & $0.009 * *$ \\
\hline MIB-1 LI > 7\% & $10.9(2.3-52.2)$ & $0.003^{* *}$ & $8.8(1.9-48.6)$ & $0.003^{* *}$ \\
\hline
\end{tabular}

PFS: progression-free survival; OS: overall survival; LI: labeling index; CI: confidence interval; * significant; ** highly significant.

TABLE 5. A Proposed Model for Estimation of Prognosis in Children with Intracranial Ependymomas

\begin{tabular}{|c|c|c|c|}
\hline \multicolumn{2}{|c|}{$\begin{array}{c}\text { Group A } \\
\text { Favorable Prognosis }\end{array}$} & \multicolumn{2}{|c|}{$\begin{array}{c}\text { Group B } \\
\text { Unfavorable Prognosis }\end{array}$} \\
\hline \multirow{3}{*}{ p53 negative and } & $\begin{array}{l}\text { MIB-1 LI }<15 \\
\text { (complete } \\
\text { resection) }\end{array}$ & \multirow{3}{*}{ p53 positive and/or } & $\begin{array}{l}\text { MIB-1 LI > } 15 \\
\text { (complete } \\
\text { resection) }\end{array}$ \\
\hline & - & & - \\
\hline & $\begin{array}{l}\text { MIB-1 LI }<5 \\
\text { (subtotal resection) }\end{array}$ & & $\begin{array}{l}\text { MIB-1 LI > } 5 \\
\text { (subtotal resection) }\end{array}$ \\
\hline
\end{tabular}

LI: labeling index.

a.

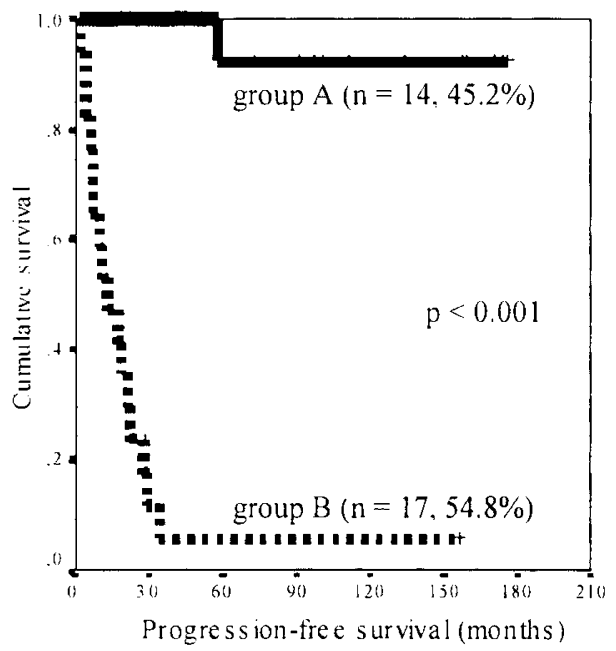

b.

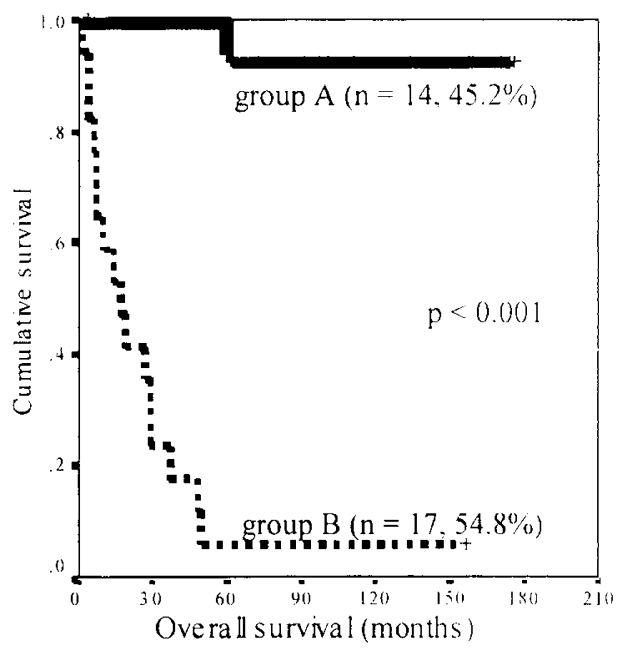

FIGURE 6. Kaplan-Meier survival curves of the ependymoma patients grouped according to the proposed prognostic scheme, see Table 5 . $P$ values of log-rank testing.

agreement in almost one third of our cases. The disagreement concerning histological diagnostic criteria of anaplastic ependymomas has been further pointed out in an extensive review of $>1400$ children with intracranial ependymomas. The incidence of anaplastic ependymomas was shown to range from $7 \%$ to $89 \%$ in 45 series summarized by the study (6).

Targeting a prognostic value of single histological criteria, the criteria of cell density of $>300$ nuclei per HPF and mitotic activity of more than seven per 10 HPF were found to confer a worse prognosis in univariate analysis in the present study. This is also supported by other investigators $(2,6,16,21)$. On the other side, we have experienced difficulty and interpersonal disagreement by a subjective assessment of hypercellularity in tumors of so-called intermediate cellularity (250-370 cells/HPF). Thus, without exact counting of the density of tumor cells, which is not optimal from the practical point of view, the evaluation of cellularity would have only a low reliability. The presence of hypercellular nodules and its close relation to a worse biological behavior demonstrated by our data has been also considered by Prayson (22). We have shown an increased proliferative activity and p53 or bcl- 2 protein positivity of cells in most of the nodules. This may indicate that such foci represent transformed subclones in the tumor with a malignant potential, and such cells may form the base for aggressive behavior. In our opinion, foci of hypercellularity should be specifically looked for in ependymomas for assessing the mitotic activity or for performing and evaluating of immunohistochemical studies.

Three immunohistochemical markers of cell proliferation (MIB-1, topo-II $\alpha$, and cyclin D1) and proliferation fractions evaluated by flow cytometry were examined in the present study. Many reports linked immunolabeling of Ki-67 antigen, which is present in all phases of the cell cycle except for G0 (23), with tumor proliferation and clinical outcome in a wide variety of malignancies, including those of 
the CNS (24). Relatively few studies have been focused on investigation of MIB-1 LI in ependymomas $(8,10,16,19,22,25-28)$. Except for two of them (22, 27), a significant association between Ki-67/ MIB-1 LI and a high tumor grade was reported. In four of the studies $(16,19,22,26)$, subgroups with most significant differences in PFS were separated by cut-off points. In the present study, the cutoff point was $7 \%$ for the entire group of patients. The variation of cut-off points in the reports $(1,9,4$, and $20 \%$, respectively) might be influenced by a small number of examined tumors, heterogeneity of studied cohorts, sampling errors, and technical details, for instance, fixation in Bouin's fixative (16). The other possible explanation is the varying proportion of patients who underwent a total surgical removal of the tumor. Our data demonstrated significantly different cut-off values of variables describing proliferative activity in these subgroups.

Topoisomerase-II $\alpha$ (topo-II $\alpha$ ) is an enzyme playing a role in the chromosome segregation and condensation, and it is expressed in cells in the S, G2 and M phases of the cell cycle (29). Some investigators studied immunoexpression of topoisomerase-II $\alpha$ in astrocytomas $(30,31)$ and showed a close correlation of topo-II $\alpha$ LI to MIB-1 LI. We demonstrated a similar finding in ependymomas. Korshunov et al. (32) suggested using antibody against topo-II $\alpha$ instead of MIB-1 to avoid the problem of varying cut-off values of MIB-1 LI and demonstrated a significant correlation between topo-II $\alpha \mathrm{LI}$ and poor prognosis with a cutoff point of $5 \%$. This is in keeping with our findings, although we identified a cutoff value of $12 \%$. In comparison with MIB-1 LI, topo-II $\alpha$ LI was demonstrated to have decreased predictive value in our series. Along with the finding of a dual pattern of topoII $\alpha$ immunostaining intensity, we would not prefer using this antibody to anti-MIB-1.

The immunoexpression of cyclin D1, which facilitates cell cycle progression through the G1 phase into the S phase of the cycle (33), has been demonstrated in various tumors. In ependymal tumors, it was only Prayson (27) who studied the potential role of cyclin D1. His finding that cyclin D1 LI did not correlate with a clinical outcome reliably is supported by our data.

Although abnormalities of the p53 gene are found in many astrocytic gliomas (34), there are only limited series investigating the immunoexpression of p53 in ependymomas $(8,28,32)$. Their common finding of a strong association with both the MIB-1 LI and the poor outcome has been reaffirmed also by or data. The overall incidence of p53 positivity in the present series was $35.5 \%$, which, together with the rate of $27 \%$ of 88 examined cases (32), contradicts the previous suggestions, that the p53 gene mutations are only rarely associated with ependymomas $(35,36)$. In the contrary, the data concern- ing immunoexpression of the p53 protein indicate its usefulness in identification of more aggressive clones in ependymomas and its superior predictive value.

Despite the fact that there is little known about mechanisms involved, expression of bcl-2 protein has been reported identified in several brain tumors (37). There has been only a single report (28) concerning the bcl-2 positivity in a limited number of ependymomas (none of 17 low-grade and 1 of 4 anaplastic were positive). Our results suggest that evaluation of bcl-2 immunoexpression is useful in identifying aggressive ependymomas. The role of caspase-3/CPP32 has been also considered in some human neoplasms $(38,39)$. To our knowledge, the present study is the first one that has specifically looked at the presence of caspase- 3 in ependymal lesions. However, our investigation showed only a low rate of caspase-3 immunoexpression (13\%) in ependymomas, without an evident association with their biological behavior or prognosis.

Some studies evaluating immunoreactivity for glial and epithelial markers (GFAP, EMA, CK) in ependymomas (40-43) suggested a link to the biological behavior. Our results indicate that evaluation of a glial or epithelial immunophenotype is of a limited use for predicting the biological behavior of these tumors.

Association of cellular DNA content and biologic behavior of pediatric ependymomas has been considered in three reports with a total of 49 cases investigated. The results were controversial. Kotylo et al. (44) observed an association of diploid DNA stemline with shortened survival in a group of 17 pediatric patients. Spaar et al. (45) found aneuploid or polyploid histograms in four anaplastic ependymomas, whereas no association between the ploidy status and the outcome of 22 patients was observed by Reyes-Mugica et al. (46). The lack of association of the ploidy and the outcome of the disease was also demonstrated in our series.

In conclusion, our analysis of prognostic factors in children with intracranial ependymomas and the review of the literature demonstrated that the conventional histological evaluation alone has a poor predictive capacity regarding the biological behavior in individual cases. Our findings suggest that the crucial point in the clinical course and outcome of children with intracranial ependymomas is the extent of the surgical resection. Thereafter the clinical outcome is critically influenced by the biological properties of the tumor determining cellular proliferation. Their estimation should lead to identification of prognostic subgroups of patients who are at risk for developing recurrent tumor or of those who might benefit from a less aggressive adjuvant therapy. From a wide spectrum of histological, immunohistochemical, and flow cytometric factors, the 
evaluation of proliferation kinetics (at best mirrored by MIB-1 LI) and p53 or bcl-2 immunoexpression was shown to have the greatest impact. Although a proposed guideline evaluating the extent of surgery, MIB-1 LI, and p53 seems to be promising, it was tested on a limited number of cases and the concept needs to be reevaluated in a larger cohort of patients, probably in a multi-institutional or international study.

Acknowledgments: The authors wish to thank Hannes Vogel, M.D., Stanford University (Stanford, CA) for his kind review of the manuscript.

\section{REFERENCES}

1. Wiestler DO, Schiffer D, Coons SW, Prayson RA, Rosenblum MK. Ependymoma. Anaplastic ependymoma. In: Kleihues P, Cavenee WK, editors. WHO classification of tumours: pathology and genetics of tumours of the nervous system. Lyon, France: IARC Press; 2000. p. 72-7.

2. Foreman NK, Love S, Thorne R. Intracranial ependymomas: analysis of prognostic factors in a population-based series. Pediatr Neurosurg 1996;24:119-25.

3. Robertson PL, Zeltzer PM, Boyett JM, Rorke LB, Allen JC, Geyer JR, et al. Survival and prognostic factors following radiation therapy and chemotherapy for ependymomas in children: a report of the Children's Cancer Group. J Neurosurg 1998;88:695-703.

4. Ross GW, Rubinstein LJ. Lack of histopathological correlation of malignant ependymomas with postoperative survival. J Neurosurg 1989;70:31-6.

5. Pollack IF, Gerszten PC, Martinez AJ, Lo KH, Shultz B, Albright AL, et al. Intracranial ependymomas of childhood: long-term outcome and prognostic factors. Neurosurgery 1995;37:655-66.

6. Bouffet E, Perilongo G, Canete A, Massimino M. Intracranial ependymomas in children: a critical review of prognostic factors and a plea for cooperation. Med Pediatr Oncol 1998; 30:319-29.

7. McLendon RE, Enterline DS, Tien RD, Thorstad WL, Bruner JM. Tumors of central neuroepitelial origin. In: Bigner DD, McLendon RE, Bruner JM, editors. Russell and Rubinstein's pathology of tumors of the nervous system. 6th ed. London: Arnold; 1998. p. 392-417.

8. Suzuki S, Oka H, Kawano N, Tanaka S, Utsuki S, Fujii K. Prognostic value of Ki-67 (MIB-1) and p53 in ependymomas. Brain Tumor Pathol 2001;18:151-4.

9. Constantini S, Houten J, Miller DC, Freed D, Ozek MM, Rorke LB, et al. Intramedullary spinal cord tumors in children under the age of 3 years. J Neurosurg 1996;85:1036-43.

10. Bennetto L, Foreman N, Harding B, Hayward R, Ironside J, Love S, et al. Ki-67 immunolabeling index is a prognostic indicator in childhood posterior fossa ependymomas. Neuropathol Appl Neurobiol 1998;24:434-40.

11. Schiffer D, Chio A, Giordana MT, Migheli A, Palma L, Pollo B, et al. Histologic prognostic factors in ependymoma. Childs Nerv Syst 1991;7:177-82.

12. Hedley DW. DNA analysis from paraffin-embedded blocks. Methods Cell Biol 1994;41:231-40.

13. Ernestus RI, Schroder R, Stutzer H, Klug N. The clinical and prognostic relevance of grading in intracranial ependymomas. Br J Neurosurg 1997;11:421-8.

14. Nazar GB, Hoffman HJ, Becker LE, Jenkin D, Humphreys RP, Hendrick EB. Infratentorial ependymomas in childhood: prognostic factors and treatment. J Neurosurg 1990;72:40817.

15. Rousseau P, Habrand JL, Sarrazin D, Kalifa C, TerrierLacombe MJ, Rekacewicz C, et al. Treatment of intracranial ependymomas of children: review of a 15-year experience. Int J Radiat Oncol Biol Phys 1994;28:381-6.

16. Figarella-Branger D, Civatte M, Bouvier-Labit C, Gouvernet J, Gambarelli D, Gentet JC, et al. Prognostic factors in intracranial ependymomas in children. J Neurosurg 2000;93:60513.

17. Sala F, Talacchi A, Mazza C, Prisco R, Ghimenton C, Bricolo A. Prognostic factors in childhood intracranial ependymomas: the role of age and tumor location. Pediatr Neurosurg 1998;28:135-42.

18. Perilongo G, Massimino M, Sotti G, Belfontali T, Masiero L, Rigobello L, et al. Analyses of prognostic factors in a retrospective review of 92 children with ependymoma: Italian Pediatric Neuro-oncology Group. Med Pediatr Oncol 1997; 29:79-85.

19. Ho DM, Hsu CY, Wong TT, Chian H. A clinicopathologic study of 81 patients with ependymomas and proposal of diagnostic criteria for anaplastic ependymoma. J Neurooncol 2001;54:77-85.

20. McLaughlin MP, Marcus RB, Buatti JM, McCollough WM, Mickle JP, Kedar A, et al. Ependymoma: results, prognostic factors and treatment recommendations. Int J Radiat Oncol Biol Phys 1998;40:845-50.

21. Schiffer D, Giordana MT. Prognosis of ependymoma. Childs Nerv Syst 1998;14:357-61.

22. Prayson RA. Clinicopathologic study of 61 patients with ependymoma including MIB-1 immunohistochemistry. Ann Diagn Pathol 1999;3:11-8.

23. Gerdes J, Li L, Schlueter C, Duchrow M, Wohlenberg C, Gerlach $\mathrm{C}$, et al. Immunobiochemical and molecular biologic characterization of the cell proliferation-associated nuclear antigen that is defined by monoclonal antibody Ki-67. Am J Pathol 1991;138:867-73.

24. Brown DC, Gatter KC. Monoclonal antibody Ki-67: its use in histopathology. Histopathology 1990;17:489-503.

25. Schroder R, Ploner C, Ernestus RI. The growth potential of ependymomas with varying grades of malignancy measured by the Ki-67 labeling index and mitotic index. Neurosurg Rev 1993;16:145-50.

26. Ritter AM, Hess KR, McLendon RE, Langford LA. Ependymomas. MIB-1 proliferation index and survival. J Neurooncol 1998;40:51-7.

27. Prayson RA. Cyclin D1 and MIB-1 immunohistochemistry in ependymomas: a study of 41 cases. Am J Clin Pathol 1998; 110:629-34.

28. Rushing EJ, Brown DF, Hladik CL, Risser RC, Mickey BE, White CL. Correlation of bcl-2, p53, and MIB-1 expression with ependymoma grade and subtype. Mod Pathol 1998;11: 464-70.

29. Camplejohn RS, Brock A, Barnes DM, Gillett C, Raikundalia B, Kreipe H, et al. Ki-S1, a novel proliferative marker: flow cytometric assessment of staining in human breast carcinoma cells. Br J Cancer 1993;67:657-62.

30. Taniguchi K, Wakabayashi T, Yoshida T, Mizuno M, Yoshikawa K, Kikuchi A, et al. Immunohistochemical staining of DNA topoisomerase IIalpha in human gliomas. J Neurosurg 1999;91:477-82.

31. Holden JA, Townsend JJ. DNA topoisomerase II-alpha as a proliferation marker in astrocytic neoplasms of the central nervous system: correlation with MIB1 expression and patient survival. Mod Pathol 1999;12:1094-100.

32. Korshunov A, Golanov A, Timirgaz V. Immunohistochemical markers for intracranial ependymoma recurrence. An analysis of 88 cases. J Neurol Sci 2000;177:72-82. 
33. Lukas J, Pagano M, Staskova Z, Draetta G, Bartek J. Cyclin D1 protein oscillates and is essential for cell cycle progression in human tumour cell lines. Oncogene 1994;9:707-18.

34. Nieder C, Petersen S, Petersen C, Thames HD. The challenge of p53 as prognostic and predictive factor in gliomas. Cancer Treat Rev 2000;26:67-73.

35. Fink KL, Rushing EJ, Schold SC, Nisen PD. Infrequency of p53 gene mutations in ependymomas. J Neurooncol 1996;27:111-5.

36. Kramer DL, Parmiter AH, Rorke LB, Sutton LN, Biegel JA. Molecular cytogenetic studies of pediatric ependymomas. J Neurooncol 1998;37:25-33.

37. Nakasu S, Nakasu Y, Nioka H, Nakajima M, Handa J. Bcl-2 protein expression in tumors of the central nervous system. Acta Neuropathol 1994;88:520-6.

38. Sohn JH, Kim DH, Choi NG, Park YE, Ro JY. Caspase-3/CPP32 immunoreactivity and its correlation with frequency of apoptotic bodies in human prostatic carcinomas and benign nodular hyperplasias. Histopathology 2000;37:555-60.

39. Izban KF, Wrone-Smith T, Hsi ED, Schnitzer B, Quevedo ME, Alkan S. Characterization of the interleukin-lbeta-converting enzyme/ced-3-family protease, caspase-3/CPP32, in Hodgkin's disease: lack of caspase-3 expression in nodular lymphocyte predominance Hodgkin's disease. Am J Pathol 1999;154:1439-47.

40. Vege KD, Giannini C, Scheithauer BW. The immunophenotype of ependymomas. Appl Immunohistochem Mol Morphol 2000;8:25-31.
41. Figarella-Branger D, Gambarelli D, Dollo C, Devictor B, Perez-Castillo AM, Genitori L, et al. Infratentorial ependymomas of childhood. Correlation between histological features, immunohistological phenotype, silver nucleolar organizer region staining values and post-operative survival in 16 cases. Acta Neuropathol (Berl) 1991;82:208-16.

42. Cruz-Sanchez FF, Rossi ML, Esiri MM, Reading M. Epithelial membrane antigen expression in ependymomas. Neuropathol Appl Neurobiol 1988;14:197-205.

43. Kaneko Y, Takeshita I, Matsushima T, Iwaki T, Tashima T, Fukui M. Immunohistochemical study of ependymal neoplasms: histological subtypes and glial and epithelial characteristics. Virchows Arch A Pathol Anat Histopathol 1990; 417:97-103.

44. Kotylo PK, Robertson PB, Fineberg NS, Azzarelli B, Jakacki R. Flow cytometric DNA analysis of pediatric intracranial ependymomas. Arch Pathol Lab Med 1997;121:1255-8.

45. Spaar FW, Blech M, Ahyai A. DNA-flow fluorescencecytometry of ependymomas. Report on ten surgically removed tumours. Acta Neuropathol (Berl) 1986;69:15360.

46. Reyes-Mugica M, Chou PM, Myint MM, Ridaura-Sanz C, Gonzalez-Crussi F, Tomita T. Ependymomas in children: histologic and DNA-flow cytometric study. Pediatr Pathol 1994;14:453-66. 